ESJ Social Sciences

\title{
Evaluation de la contribution de l'irrigation à la croissance économique et agricole : cas du Maroc
}

\author{
Fatima Ezzahra Mengoub, Doctorante
}

Institut Agronomique et vétérinaire Hassan II, Rabat, Maroc

Caroline Lejars, Dr.

Centre de coopération internationale en recherche agronomique pour le développement, France

Mohammed Rachid Doukkali, Pr

Institut Agronomique et vétérinaire Hassan II, Rabat, Maroc

\section{Doi:10.19044/esj.2021.v17n27p218}

Submitted: 26 July 2021

Accepted: 27 August 2021

Published: 31 August 2021
Copyright 2021 Author(s)

Under Creative Commons BY-NC-ND

4.0 OPEN ACCESS

Cite As:

Mengoub F. E., Lejars C. \& Doukkali M. R. (2021). Evaluation de la contribution de l'irrigation à la croissance économique et agricole : cas du Maroc. European Scientific Journal, ESJ, 17 (27), 218. https://doi.org/10.19044/esj.2021.v17n27p218

\section{Résumé}

Situé dans une zone géographique où les ressources hydriques sont limitées, le Maroc a multiplié les efforts pour gérer ses ressources en eau. Ces efforts se sont concrétisés à travers l'amélioration continue du cadre législatif régissant le secteur de l'eau et s'adaptant aux nouvelles contraintes qu'elles soient climatiques, technologiques et/ou économiques. Dans ce sens, ce travail vise l'évaluation de l'impact de ces interventions publiques sur la croissance agricole et économique observées. La démarche méthodologique adoptée se base sur la méthode de comptabilité de croissance ou " Growth Accounting », développée par Solow en 1957. Elle permet de décomposer la croissance en contribution des facteurs et celle de la productivité. Les résultats de cette étude confirment le rôle primordial que joue l'agriculture en tant que moteur de croissance de l'économie marocaine. Ils montrent également que la contribution de l'eau à la croissance de la valeur ajoutée agricole est de l'ordre de $2 \%$ et qu'elle demeure tributaire des conditions climatiques, qui à leur tour changent sous l'effet du réchauffement climatique. 
Mots clés : Maroc, irrigation, comptabilité de la croissance, productivité totale des facteurs, agriculture, eau

\title{
Evaluating the contribution of irrigation to economic and agricultural growth: the case of Morocco
}

\author{
Fatima Ezzahra Mengoub, Doctorante \\ Hassan II Agronomic and Veterinary Institute, Rabat, Morocco \\ Caroline Lejars, Dr. \\ Center of International Cooperation in Agricultural Research for \\ Development, France \\ Mohammed Rachid Doukkali, Pr \\ Hassan II Agronomic and Veterinary Institute, Rabat, Morocco
}

\begin{abstract}
Located in a geographical area with limited water resources, Morocco has multiplied its efforts to manage its water resources. These efforts have been materialized through the continuous improvement of the legislative framework governing the water sector and adapting to the new constraints whether they are climatic, technological and/or economic. In this sense, this work aims at evaluating the impact of these public interventions on the observed agricultural and economic growth. The methodological approach adopted is based on the growth accounting method developed by Solow in 1957. It allows for the decomposition of growth into factor contributions and productivity contributions. The results of this study confirm the primary role played by agriculture as an engine of growth in the Moroccan economy. They also show that the contribution of water to the growth of agricultural value added is around $2 \%$ and that it remains dependent on climatic conditions which are changing under global warming.
\end{abstract}

Keywords: Morocco, irrigation, growth accounting, total factor productivity, agriculture, water

\section{Introduction}

Les changements technologiques et institutionnels sont considérés comme étant des facteurs clés pour atteindre les niveaux de croissance espérés (Solow, 1956 ; North, 1990 ; Acemoglu, 2002 ; Zaman et Goschin, 2010). En effet, ils viennent compenser la rareté d'un ou de plusieurs facteurs de production dits limitants. Cette compensation permet aux économies d'enregistrer des croissances ininterrompues et considérables en dépit des contraintes techniques ou structurelles auxquelles elles font face (Acemoglu 
et al., 2004). Les facteurs limitants diffèrent d'un pays à un autre et dépendent de plusieurs caractéristiques intrinsèques, telles que les dotations en ressources, le taux de croissance démographique ou la qualité du capital humain.

Situé dans une zone géographique où les ressources hydriques se font de plus en plus rares, le Maroc figure parmi les pays les plus menacés par une pénurie d'eau. Selon le dernier rapport sur l'état du stress hydrique global, le World Resources Institute (WRI) a classé le Maroc au $22^{\text {ième }}$ rang des pays les plus exposés aux risques d'insécurité hydrique (WRI, 2019). En effet, les ressources en eau au Maroc subissent différentes pressions à savoir l'augmentation de la demande en eau en raison de l'explosion démographiques, les sécheresses récurrentes et l'irrégularité des précipitations dus aux effets du changement climatique et enfin les exigences du développement économiques. En raison de ces différentes pressions, les disponibilités en eau par habitant sont passées de plus de $2500 \mathrm{~m}^{3}$ par habitant par an en 1960 à moins de $700 \mathrm{~m}^{3}$ par habitant par an en 2019, soit une baisse de $75 \%$ (DEPF, 2020).

En plus des dotations en eau limitées, les ressources en eau mobilisables au Maroc sont variables dans le temps et dans l'espace (Agoumi, 2006). Le Maroc compte environ 14 grands bassins hydrographiques contrastés en termes de dotation en eau. Les précipitations reçues en amont de tous ces bassins totalisent environ 19,3 milliards de $\mathrm{m}^{3}$, dont seuls 17,7 milliards de $\mathrm{m}^{3}$ sont mobilisables (Mili, 2020). A eux seuls, les bassins nordiques, rifains et ceux situés dans le centre du pays fournissent environ $72 \%$ des eaux mobilisables tandis que les bassins hydrographiques du sud ne contribuent qu'à hauteur de 5\% des eaux mobilisables (MEME, 2010). Quant aux ressources hydriques souterraines, le potentiel exploitable des eaux de nappes est estimé à 4 milliards de $\mathrm{m}^{3}$ par an (Hssaisoune et al., 2020).

Face à la rareté de l'eau, l'Etat Marocain a multiplié les efforts pour gérer ses ressources hydriques. Ces efforts consistent en l'amélioration progressive du dispositif législatif qui régit le secteur de l'eau et en l'adaptation aux nouvelles contraintes, climatiques, technologiques et/ou économiques (Bouderbala, 1999 ; Kleiche, 200 ; Aspe et al, 2012 ; Doukkali et Grijsen, 2018). En effet, depuis l'indépendance, le Maroc a beaucoup investi pour développer un patrimoine hydrique important, constitué aujourd'hui d'une centaine de barrages et d'infrastructures de base pour satisfaire les besoins hydriques du pays, en particulier ceux de l'agriculture irriguée (Ezzahra Mengoub et al, 2021).

Le développement de l'irrigation a induit l'introduction de nouvelles techniques et technologies de production ayant pour but l'amélioration de la productivité agricole. L'adoption des avancées technologiques en matière d'irrigation a permis l'intensification de la production agricole de façon 
considérable, permettant ainsi au secteur agricole de réaliser des taux de croissance importants et une meilleure réallocation des ressources (Sadiki, 2017). Quant à la gouvernance de l'eau, elle est restée au centre des stratégies élaborées par les pouvoirs publics et a permis la mise en place de plusieurs institutions gérant toutes les parties prenantes et assurant également le suivi de l'offre et de la demande en eau. De plus, elle a permis de promulguer des codes et des lois régissant le secteur de l'eau pour contrôler et rationnaliser son utilisation (Molle et al, 2019).

Bien que les terres irriguées ne représentent que 20\% des superficies assolées, l'agriculture irriguée au Maroc contribue aujourd'hui à hauteur de $45 \%$ de la valeur ajoutée agricole et représentent $75 \%$ des exportations agricoles totales (Harbouze et al., 2019). L'irrigation a également permis aux agriculteurs marocains de diversifier leur production et de pratiquer des cultures plus valorisante de l'eau. Enfin, elle a permis à l'agriculture de devenir de plus en plus résiliente, surtout face aux aléas climatiques (Sadiki, 2017).

Les réformes institutionnelles et les changements technologiques induits par l'irrigation sont importants et l'expérience marocaine offre des enseignements remarquables en termes de politiques de gestion de ressources hydriques (Doukkali, 2005). L'objectif de cette étude est d'évaluer la contribution des changements technologique et institutionnel induits par l'irrigation sur la croissance agricole et économique au Maroc. En d'autres termes, cet article vise à évaluer l'impact de toutes les interventions publiques en matière de structuration du secteur irrigué sur la productivité du secteur agricole ainsi que sur la croissance agricole et économique.

\section{Approche méthodologique}

Pour évaluer la part de la croissance associée à l'augmentation de l'utilisation des facteurs utilisés, et celle due au progrès technologique et au changement institutionnel, ce travail s'appuie sur la méthode de comptabilité de croissance ou "Growth accounting », développée par Solow en 1956. Cette méthode mesure la contribution des différents facteurs de production dans la croissance observée et attribue la part de la croissance qui ne peut être expliquée par l'augmentation de l'utilisation des facteurs à l'amélioration de la productivité $\left(\mathrm{PTF}^{1}\right)$.

Le recours à la méthode de comptabilité de croissance requiert une analyse approfondie et une connaissance détaillée des facteurs étudiés (Welhan, 2020). Des séries détaillant l'évolution de l'utilisation des facteurs en volume doivent être prises en considération afin de neutraliser les effets des variations des prix. La méthode exige également l'utilisation des stocks des

\footnotetext{
${ }^{1} \mathrm{PTF}$ : productivité totale des facteurs
} 
facteurs de production. Pour le facteur capital, la comptabilité nationale ne tient compte que des flux des investissements (formation brute du capital fixe), d'où la nécessité d'estimer le stock du capital économique. Pour ce faire, le présent travail se base sur la méthode de l'inventaire permanent, expliquée dans la section suivante.

\section{1. Évaluation du stock du capital physique}

La méthode de l'inventaire permanent (MIP) est l'approche la plus utilisée pour mesurer le stock du capital dans une économie donnée (Ahmed, 2008). Cette méthode repose sur une hypothèse principale qui suppose que le stock du capital est constitué par le flux des investissements cumulés et corrigés par les retraits des pertes et des amortissements du stock existant (OCDE, 2009). Les données utilisées dans cette méthode sont les investissements approchés par la Formation Brute du Capital Fixe (FCBC) et le taux de dépréciation permettant de soustraire la part du stock déjà amortie. Pour illustrer cette méthode, il faut considérer l'équation suivante :

$$
\begin{gathered}
K_{\tau+1}=(1-\delta) k_{\tau}+I_{t} \\
\text { Avec : } \\
K_{\tau+1}: \text { est le stock du capital à l'instant } \mathrm{t}+1 \\
I_{t} \quad: \text { est l'investissement à l'instant } \mathrm{t} \\
\begin{array}{c}
\tau \\
\delta \quad \text { la période étudiée } 1,2,3, \ldots, t \\
\quad: \text { est le taux de dépréciation }
\end{array}
\end{gathered}
$$

Pour la première année, $\tau=1, K_{1}$ peut s'écrire sous la forme suivante :

$$
K_{1}=(1-\delta) k_{0}+I_{0}
$$

Ainsi pour la deuxième année $K_{2}$ est égale à :

$$
K_{2}=(1-\delta) k_{1}+I_{2}
$$

Remplacer $K_{1}$ par sa valeur, l'équation (3) prend la forme suivante :

$$
K_{2}=\left[(1-\delta)^{2} k_{0}+(1-\delta) I_{1}\right]+I_{2}
$$

Sachant que cette étude considère 37 années (1981-2017), par analogie, l'équation (4) devient :

$$
K_{37}=(1-\delta)^{37} k_{0}+(1-\delta)^{36} I_{0}+\underset{(1-\delta)^{35} \cdot I_{1}+(1-\delta)^{34} \cdot I_{2}+\cdots+(1-\delta) \cdot I_{35}+}{I_{36}}
$$

Plus généralement, cette équation peut être écrite sous la forme suivante

$$
K_{\tau 0+\tau *}=(1-\delta)^{\tau *} k_{0}+\sum_{t=1}^{\tau *}(1-\delta)^{\tau *-t} I_{\tau 0+t-1}
$$


D'un autre côté, diviser cette dernière équation par $K_{0}$, donne l'équation suivante :

$$
\frac{K_{\tau 0+\tau *}}{K_{0}}=(1-\delta)^{\tau *}+\frac{\sum_{t=1}^{\tau *}(1-\delta)^{\tau *-t} I_{\tau 0+t-1}}{K_{0}}
$$

Sachant que :

$$
\begin{aligned}
\frac{K_{\tau 0+1}}{K_{\tau 0}} & =\left(\frac{K_{\tau 0+\tau *}}{K_{\tau 0}}\right)^{\frac{1}{\tau^{*}}} \\
\text { Pour } \tau_{0} & =0 \text { et } \tau^{*}=37 \\
\frac{K_{1}}{K_{0}} & =\left(\frac{K_{37}}{K_{0}}\right)^{\frac{1}{37}}
\end{aligned}
$$

Ainsi remplacer $K_{37}$ par sa valeur donne l'équation suivante:

$$
\frac{K_{1}}{K_{0}}=\left(\frac{(1-\delta)^{37} k_{0}+(1-\delta)^{36} I_{0}+(1-\delta)^{35} \cdot I_{1}+\cdots+(1-\delta) \cdot I_{35}+I_{36}}{K_{0}}\right)^{\frac{1}{37}}
$$

D'où :

$$
\frac{K_{1}}{K_{0}}=\left((1-\delta)^{37}+\frac{(1-\delta)^{36} I_{0}+(1-\delta)^{35} \cdot I_{1}+\cdots+(1-\delta) \cdot I_{35}+I_{36}}{K_{0}}\right)^{\frac{1}{37}}
$$

Or, sachant que :

$$
\begin{gathered}
\frac{K_{1}}{K_{0}}=(1-\delta)+\frac{I_{0}}{K_{0}} \\
\text { Donc : } \\
(1-\delta)+\frac{I_{0}}{K_{0}}=\left((1-\delta)^{37}+\frac{(1-\delta)^{36} I_{0}+(1-\delta)^{35} \cdot I_{1}+\cdots+(1-\delta) \cdot I_{35}+I_{36}}{K_{0}}\right)^{\frac{1}{37}}
\end{gathered}
$$

Pour le cas général :

$$
(1-\delta)+\frac{I_{\tau 0}}{k_{\tau 0}}=\left[(1-\delta)^{\tau *}+\frac{\sum_{t=1}^{\tau *}(1-\delta)^{\tau *-t} I_{\tau 0+t-1}}{K_{\tau 0}}\right]^{\frac{1}{\tau} *}
$$

À ce stade, l'équation ci-dessous contient deux inconnues à savoir le stock initial quand $\tau=0$ et le taux de dépréciation $\delta$, tandis que $I_{t}$ est la formation brute du capital fixe au moment t. En ce qui concerne le taux de dépréciation, plusieurs chercheurs ont essayé de le calculer, notamment Nehru 
et Dhareshwar (1993), Bu Y. (2004) et Baldwin J. et al. (2005). Le taux de dépréciation du stock du capital dépend de plusieurs caractéristiques intrinsèques à chaque économie. L'absence de données fiables, surtout pour les économies en voie de développement, fait que les économistes supposent que généralement la valeur du taux de dépréciation du stock du capital physique est de l'ordre de 4\% (Baldwin, 2005). En parallèle, d'autres économistes ont tenté de calculer ce taux dans plusieurs économies asiatiques à savoir la Corée du Nord, les Philippines, et l'Indonésie et dans quelques économies africaines, notamment la Côte d'Ivoire, le Ghana et le Kenya (Bu, 2004). Les résultats de cette étude montrent que les taux de dépréciation diffèrent d'une économie à une autre et sont nettement supérieurs à la moyenne prise comme référence dans la littérature.

En l'absence de données fiables, ce travail suppose que le taux de dépréciation du capital fixe dans l'économie marocaine est de l'ordre de 0.04 (HCP, 2005). Suite à cette hypothèse, l'équation (14) aura une seule variable inconnue qui est le stock du capital initial. La résolution du problème est désormais possible par le recours à une méthode basée sur la résolution d'un problème mathématique de programmation non-linéaire (Huard, 1971). Dans cette étude, la résolution de ce problème indique que le stock du capital physique au niveau de l'économie marocaine est estimé, en début de 1981, à 653 milliards de $2007 \mathrm{MAD}^{2}$. Il est passé à 3377 milliards de MAD en 2017, soit une augmentation d'environ 4.5\% (Figure 1).

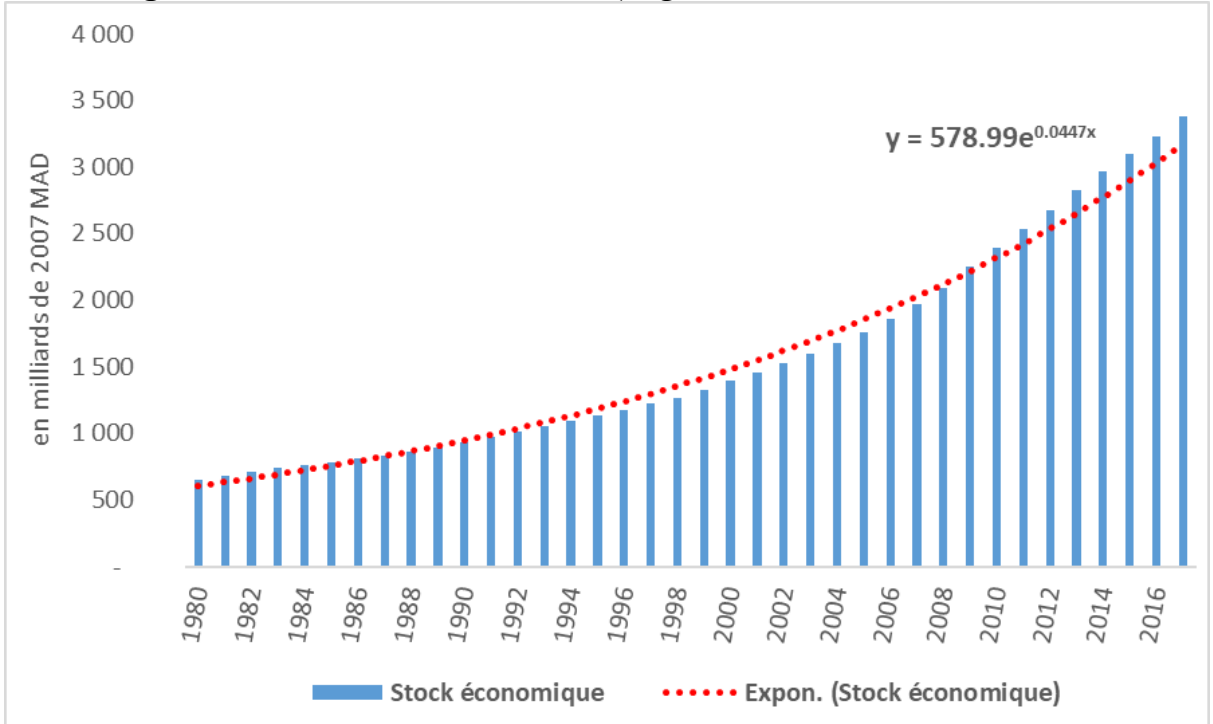

Figure 1. Evolution de la valeur du stock du capital physique au Maroc sur la période 19812017 en dirhams 2007

${ }^{2}$ MAD : Dirham marocain 


\subsection{Désagrégation du stock du capital par secteur}

Après avoir estimé le stock du capital physique dans toute l'économie marocaine, l'étape suivante est la répartition de ce stock par secteur économique afin d'évaluer la PTF dans le secteur agricole et dans le reste de l'économie. La méthode de base adoptée pour la désagrégation du stock du capital par secteur est celle développée par Roe et Smith (2014). Cette méthode calcule le coût implicite du capital $\left(C_{K}\right)$ tout en utilisant la contribution du facteur capital dans la valeur ajoutée créée par secteur suivant la formule suivante :

Où :

$$
\mathrm{C}_{\mathrm{K}}=\frac{\left(\mathrm{S}_{\mathrm{K}, \mathrm{A}} \times \mathrm{VA}_{\mathrm{A}}+\mathrm{S}_{\mathrm{K}, \mathrm{ROE}} \times \mathrm{VA}_{\mathrm{ROE}}\right)}{\mathrm{K}_{\mathrm{E}}}
$$

$S_{K, A}, S_{K, R O E}$ : sont respectivement les contributions du facteur capital dans les valeurs ajoutées générées dans le secteur agricole et les autres secteurs $\mathrm{ROE}^{3}$ (industrie et service) agrégés.

$V A_{A}, V A_{R O E}$ : valeur ajoutée agricole et celle du reste de l'économie $K_{E} \quad:$ le stock du capital dans toute l'économie

L'estimation du coût implicite du capital permet d'attribuer une valeur moyenne du capital dans l'économie. Une fois obtenue, cette valeur permet de désagréger le stock du capital par secteur en faisant recours aux valeurs ajoutées. Cette répartition est réalisée grâce à l'équation suivante :

$$
\mathrm{K}_{\mathrm{i}}=\frac{\mathrm{S}_{\mathrm{K}, \mathrm{i}} \times \mathrm{VA}_{\mathrm{i}}}{\mathrm{C}_{\mathrm{k}}}
$$

Pour l'économie marocaine, les parts du capital dans la valeur ajoutée par secteur sont extraites à partir de la matrice de comptabilité sociale de 2009 et mise à jour en 2011 par Doukkali (2011). D'après cette matrice, le capital contribue à hauteur $62 \%$ dans la valeur ajoutée agricole, $65 \%$ dans la valeur ajoutée pour les autres secteurs (service et industrie)

L'estimation du stock du capital par secteur montre que la répartition dudit stock suit la distribution suivante (Figure 2).

- $\quad 16 \%$ dans le secteur agricole

- $\quad 84 \%$ dans le reste de l'économie

${ }^{3}$ ROE : reste de l'économie 


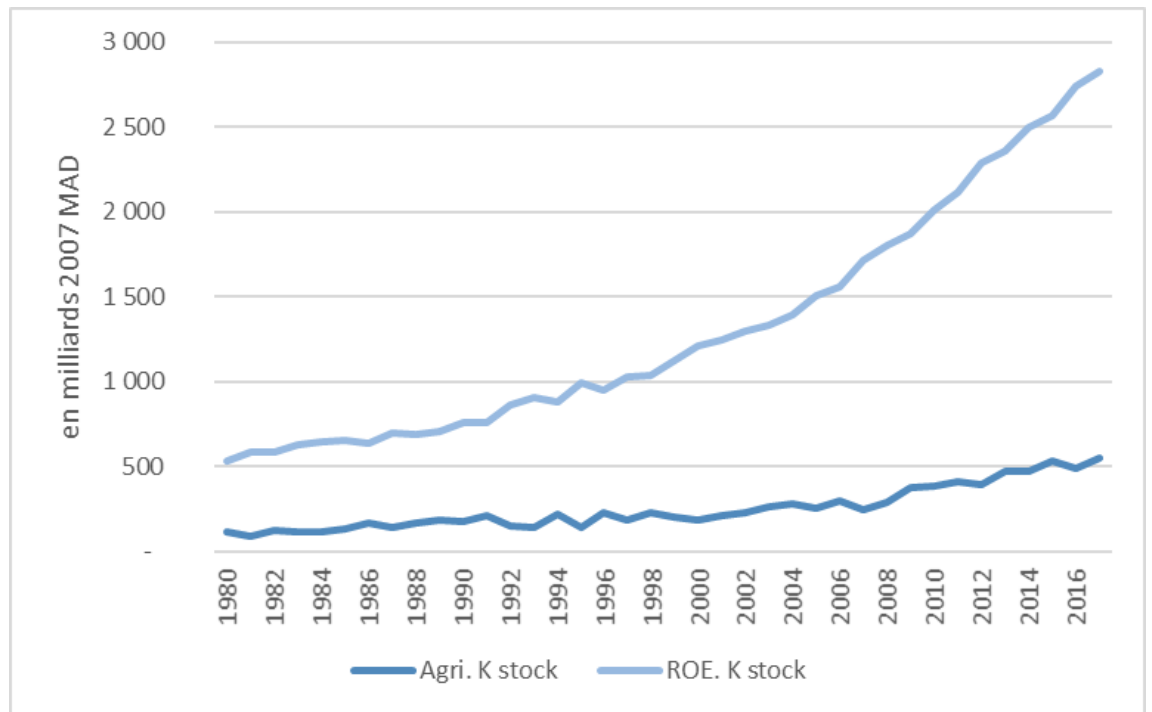

Figure 2. Répartition du Stock du capital économique dans l'agriculture et dans le reste de l'économie sur la période 1980-2017

\subsection{Approche de la comptabilité de croissance pour l'estimation de la} productivité globale des facteurs de production

L'approche méthodologique se base sur la méthode de comptabilité de croissance. Il s'agit d'une méthode qui explique la croissance économique en la décomposant en deux parties (Solow, 1956). La première partie explique la croissance de la production qui peut être attribuée à la croissance de tous les facteurs de production, tout en maintenant la technologie constante. La deuxième partie est la croissance qui est due à l'amélioration technologique et du cadre institutionnel (PTF).

La fonction de production adoptée est celle d'une fonction CobbDouglas, où quatre facteurs sont pris en considération, notamment la terre, l'eau, le travail et le capital :

$$
Y_{t}=A_{t} F\left(K_{t}, L_{t}, H_{t}, W_{t}\right)
$$

La dérivée de premier ordre donne :

$$
\begin{aligned}
& \frac{\dot{Y_{t}}}{Y_{t}}=\frac{\dot{A_{t}}}{A_{t}}+\frac{A_{t} F_{k}\left(K_{t}, L_{t}, H_{t}\right) K_{t}}{Y_{t}} \times \frac{\dot{K_{t}}}{K_{t}}+\frac{A_{t} F_{l}\left(K_{t}, L_{t}, H_{t}\right) L_{t}}{Y_{t}} \times \frac{\dot{L_{t}}}{L_{t}}+\frac{A_{t} F_{H}\left(\mathrm{~K}_{t}, L_{t}, H_{t}\right) H_{t}}{Y_{t}} \times \\
& \frac{\dot{\mathrm{H}}_{\mathrm{t}}}{\mathrm{H}_{\mathrm{t}}}+\frac{\mathrm{A}_{\mathrm{t}} \mathrm{F}_{\mathrm{W}}\left(\mathrm{K}_{\mathrm{t}}, \mathrm{L}_{\mathrm{t}}, \mathrm{H}_{\mathrm{t}}\right) \mathrm{W}_{\mathrm{t}}}{\mathrm{Y}_{\mathrm{t}}} \times \frac{\dot{\mathrm{W}}_{\mathrm{t}}}{\mathrm{W}_{\mathrm{t}}} \\
& \text { Où : } \\
& \begin{array}{c}
\frac{\dot{Y}_{t}}{Y_{t}} \quad: \text { est le taux de croissance économique } \\
\frac{\dot{A}_{t}}{A_{t}} \quad: \text { est l'amélioration de la productivité totale des facteurs } \\
\frac{A_{t} F_{k}\left(K_{t}, L_{t}, H_{t}\right) K_{t}}{Y_{t}}: \text { est la part du capital } \alpha_{k} \\
\frac{A_{t} F_{l}\left(K_{t}, L_{t}, H_{t}\right) L_{t}}{Y_{t}}: \text { est la part du travail } \alpha_{L}
\end{array}
\end{aligned}
$$


La PTF peut donc être déduite comme suit :

$$
\begin{gathered}
\frac{A_{t} F_{H}\left(K_{t}, L_{t}, H_{t}\right) H_{t}}{Y_{t}} \text { : est la part de la terre } \alpha_{H} \\
\frac{A_{t} F_{W}\left(K_{t}, L_{t}, H_{t}\right) W_{t}}{Y_{t}} \text { : est la part de l'eau d'irrigation } \alpha_{W}
\end{gathered}
$$

En remplaçant ces parts, la première dérivée prend la forme suivante :

$$
\frac{\dot{Y}_{t}}{Y_{t}}=\frac{\dot{A}_{t}}{A_{t}}+\alpha_{k} \frac{\dot{K}_{t}}{K_{t}}+\alpha_{L} \frac{\dot{L}_{t}}{L_{t}}+\alpha_{H} \frac{\dot{H}_{t}}{H_{t}}+\alpha_{W} \frac{\dot{W}_{t}}{W_{t}}
$$

$$
P T F=\frac{\dot{Y}_{t}}{Y_{t}}-\alpha_{k} \frac{\dot{K}_{t}}{K_{t}}-\alpha_{L} \frac{\dot{L_{t}}}{L_{t}}-\alpha_{H} \frac{\dot{H}_{t}}{H_{t}}-\alpha_{W} \frac{\dot{W}_{t}}{W_{t}}
$$

\section{Analyse des données}

Les données utilisées pour conduire cette évaluation sont les suivantes :

- Les données issues de la comptabilité nationale, notamment le PIB courant et constant ${ }^{4}$, la valeur ajoutée de l'agriculture et du reste de l'économie ainsi que la formation brute de capital fixe (FBCF). Ces données sont obtenues directement à partir de la base de données du Haut-commissariat au plan pour les années 1980 à 2017.

- La population active occupée pour l'ensemble de l'économie et par secteur. Les données sur la population active occupée de 1999 à 2017 ont été obtenues à partir de l'enquête nationale annuelle sur l'emploi (HCP, 2000 à 2017), de l'enquête sur l'emploi urbain de 1987 et de l'enquête sur l'emploi rural de 1986-1987. Pour compléter les séries chronologiques sur l'emploi pour l'ensemble de l'économie et par secteur, un taux de croissance de la population active occupée a été estimé sur la base des valeurs de 1987 et 1999 en utilisant une fonction exponentielle :

$$
L_{1999}=L_{1987} e^{r t}
$$

Où $L_{1999}$ et $L_{1987}$ sont la population active occupée de 1987 et 1999, t est l'année, et $\mathrm{r}$ est le taux de croissance de la population active occupée.

- Les terres agricoles : les superficies agricoles utiles cultivées sont obtenues à partir de la base de données de la FAO, depuis 1980 jusqu'à 2017. Ces valeurs prennent en considération l'augmentation des terres cultivées dans le temps.

- Les quantités d'eau d'irrigation: il s'agit des volumes d'eau superficielle et souterraine destinés à l'irrigation. Les quantités issues des barrages sont obtenues des enquêtes effectuées auprès du ministère de l'agriculture. Faute de disponibilité des données, les quantités d'eau pompées pour l'irrigation sont estimées en faisant recours au taux de croissance des terres irriguées. L'année de référence est 2011 où les quantités d'eau pompées pour des fins agricoles était de 4,2 milliards

${ }^{4}$ Les valeurs en volume considèrent l'utilisation des pris chaînés base 2007 
de $\mathrm{m}^{3}$ (SCEE, 2011). Ensuite, la série est estimée en faisant recours au taux de croissance des terres équipées en systèmes d'irrigation, utilisé dans cette étude comme proxy au taux de croissance des eaux souterraines. Les valeurs des superficies des terres équipées en systèmes d'irrigation sont extraites à partir de la base de données de la FAO.

- Parts des contributions des facteurs de production au PIB et aux valeurs ajoutées sectorielles : Les parts des facteurs capital et travail dans l'économie et par secteur sont obtenues à partir de la matrice de comptabilité sociale (MCS) nationale pour 2009, mise à jour par les auteurs pour 2011 (Doukkali, 2011). La contribution de l'eau a été estimée grâce au calcul du prix économique des ressources en eau superficielle dans la région de Tadla-Azilal. Ce prix est ensuite utilisé pour isoler la part de la contribution des ressources hydriques, souvent associée à la valeur de la terre, dans la valeur ajoutée agricole nationale et dans le PIB. Les résultats sont donnés dans le tableau 1, ci-après.

Tableau 1. contribution des facteurs de production à la valeur ajoutée par secteur et au PIB

\begin{tabular}{|c|c|c|c|c|}
\hline & Terre & Eau & Travail & Capital \\
\hline PIB & 0,2 & 0,01 & 0,35 & 0,62 \\
\hline Agriculture & 0,12 & 0,05 & 0,16 & 0,67 \\
\hline ROE & - & - & 0,35 & 0,65 \\
\hline
\end{tabular}

Source : nos calculs, sur la base de la MCS de 2011

\section{Résultats et discussion}

L'analyse de l'évolution du PIB montre que celui-ci a enregistré, sur la période 1980-2017, un taux de croissance d'environ 4,2\%. Durant cette période, le secteur agricole a augmenté en moyenne de $6,1 \%$ alors que la valeur ajoutée générée par le reste de l'économie a augmenté d'environ 3,7\%. Ceci confirme le rôle capital que joue le secteur agricole dont la croissance a été nettement supérieure à celui des autres secteurs. La figure 3 traduit cette évolution entre 1980 et 2017. 


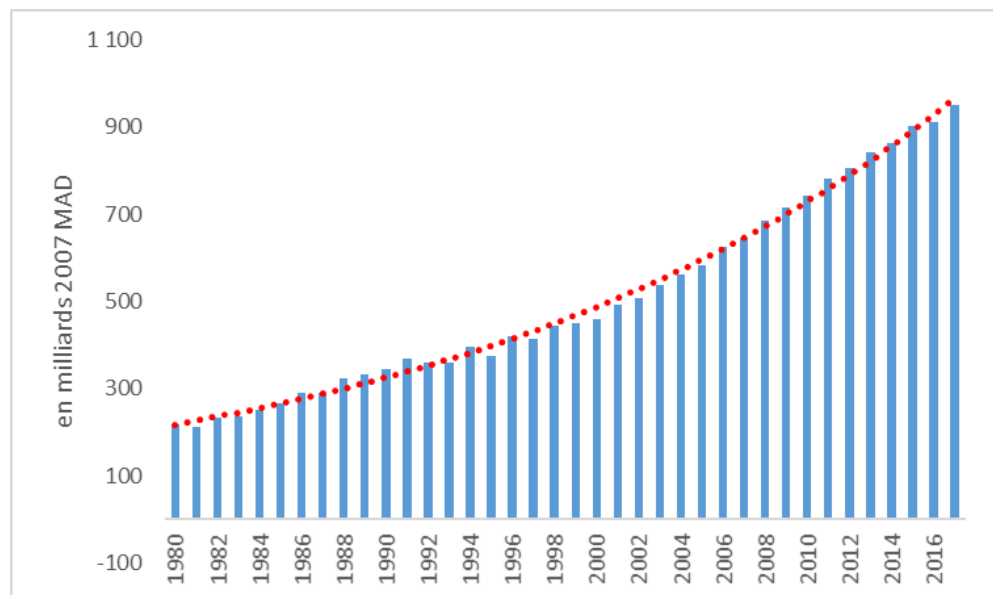

Figure 3 : Evolution du PIB en millions de dirhams sur la période 1980-2017 - prix chainés base 2007

Source : HCP,2019

La croissance économique marocaine a été tirée globalement par le capital. Ce dernier a enregistré durant la même période un taux de croissance supérieur à tous les autres facteurs, soit $4,5 \%$ contre seulement $1,1 \%$ pour la force du travail, $0,5 \%$ pour les terres agricoles et $2,8 \%$ pour l'eau (tableau 2 ). La croissance de la productivité totale des facteurs dans l'ensemble de l'économie marocaine n'a été que de $0,9 \%$ sur toute la période étudiée. Par ailleurs, le tableau 3 montre qu'environ $68 \%$ de la croissance économique au Maroc a été expliquée par l'intensification du capital. En raison du taux de croissance de la population active employée relativement faible, la contribution du facteur travail à la croissance économique n'a été que de $9 \%$.

Tableau 2. Evolution des facteurs de production, du PIB et des valeurs ajoutées sectorielles

\begin{tabular}{|c|c|c|c|c|c|c|}
\hline $\mathbf{1 9 8 0 - 2 0 1 7}$ & $\begin{array}{c}\text { Taux de } \\
\text { croissance }\end{array}$ & Eau & Terre & Travail & Capital & PTF \\
\hline PIB & 4,2 & 2,8 & 0,5 & 1,1 & 4,5 & 0,9 \\
\hline Agriculture & 6,2 & 2,8 & 0,5 & $-0,3$ & 6,2 & 1,8 \\
\hline ROE & 3.7 & & & 2,3 & 4.6 & $-0,1$ \\
\hline
\end{tabular}

Source : nos calculs 
Tableau 3. Contribution des facteurs de production dans la croissance économique et la croissance sectorielle en $\%$

\begin{tabular}{|c|c|c|c|c|c|c|}
\hline $\mathbf{1 9 8 0 - 2 0 1 7}$ & $\begin{array}{c}\text { Taux de } \\
\text { croissance }\end{array}$ & Eau & Terre & Travail & Capital & PTF \\
\hline PIB & 100 & 0,7 & 0,2 & 9,1 & 67,9 & 22,1 \\
\hline Agriculture & 100 & 2,1 & 0,9 & $-0,8$ & 69,1 & 29,9 \\
\hline ROE & 100 & & & 22 & 82 & -4 \\
\hline
\end{tabular}

Source : nos calculs

$\mathrm{Au}$ niveau sectoriel, la croissance du secteur agricole a été majoritairement expliquée par la croissance du capital qui a accusé un taux de croissance de 6,2\%, contribuant ainsi à plus de 69\% de la valeur ajoutée agricole. En effet, sur la même période, l'évolution du ratio stock du capital physique par travailleur dans le secteur agricole a augmenté d'une manière très significative en passant de 28605 MAD par travailleur à plus de 152000 MAD par travailleur en 2017, au prix de 2007. Cette évolution indique que l'accumulation du capital au niveau du secteur agricole se fait de manière plus rapide et que le secteur agricole est en train de libérer la main d'œuvre. En effet, la croissance de la population active employée dans le secteur agricole a été négative, enregistrant en moyenne une baisse de $0,8 \%$. (Tableau 1 )

L'accumulation du capital dans le secteur agricole n'est pas le fruit du hasard. Elle s'explique principalement par les nombreuses incitations accordées aux producteurs par les différentes politiques agricoles conduites dans le pays. Ces incitations ont pris différentes formes, notamment la défiscalisation du secteur et les subventions accordées à l'investissement (MAPMDREF,2013), en particulier celles dédiées au développement du secteur irrigué. Suite à ces investissements et en raison de l'intensification, les quantités d'eau destinées à l'irrigation ont augmenté significativement durant la période étudiée. En effet, celles-ci ont enregistré un taux de croissance de l'ordre de 2,8\% contribuant ainsi à environ 2,1\% de la croissance de la valeur ajoutée agricole. Cette période a été également marquée par une augmentation timide des superficies cultivées. Ainsi, les terres agricoles ont enregistré une légère augmentation de $0,5 \%$, ce qui s'est traduit par une contribution de l'ordre de $1 \%$ à la croissance de la valeur ajoutée agricole.

Les mêmes dynamiques ont été enregistrées au niveau des deux secteurs «ROE » (industriel et des services), où le capital a joué un rôle primordial dans la croissance enregistrée. Le stock du capital économique dans le reste de l'économie a augmenté d'environ 4,6\% contribuant ainsi à $82 \%$ de la croissance de la valeur ajoutée non agricole. En parallèle, la libération de la main d'œuvre agricole a été accompagnée par une augmentation de l'emploi dans les autres secteurs, en particulier dans le 
secteur des services. En effet, le nombre de travailleurs dans le secteur industriel et celui des services a augmenté de 2,3\%, expliquant ainsi $22 \%$ de la croissance de la valeur ajoutée.

En ce qui concerne l'amélioration de la productivité, le recours aux nouvelles techniques de production et l'amélioration du cadre institutionnel dans le secteur agricole ont été plus importants que dans les autres secteurs. La PTF a augmenté de 1,8\% dans l'agriculture, contribuant ainsi à environ $30 \%$ à la croissance de la valeur ajoutée agricole. Toutefois, la PTF a été négative dans les autres secteurs $(-0,3 \%)$ indiquant ainsi que la croissance au niveau de ces deux secteurs se base sur l'utilisation de plus de facteurs de production plutôt que l'amélioration de leur utilisation (figure 4).

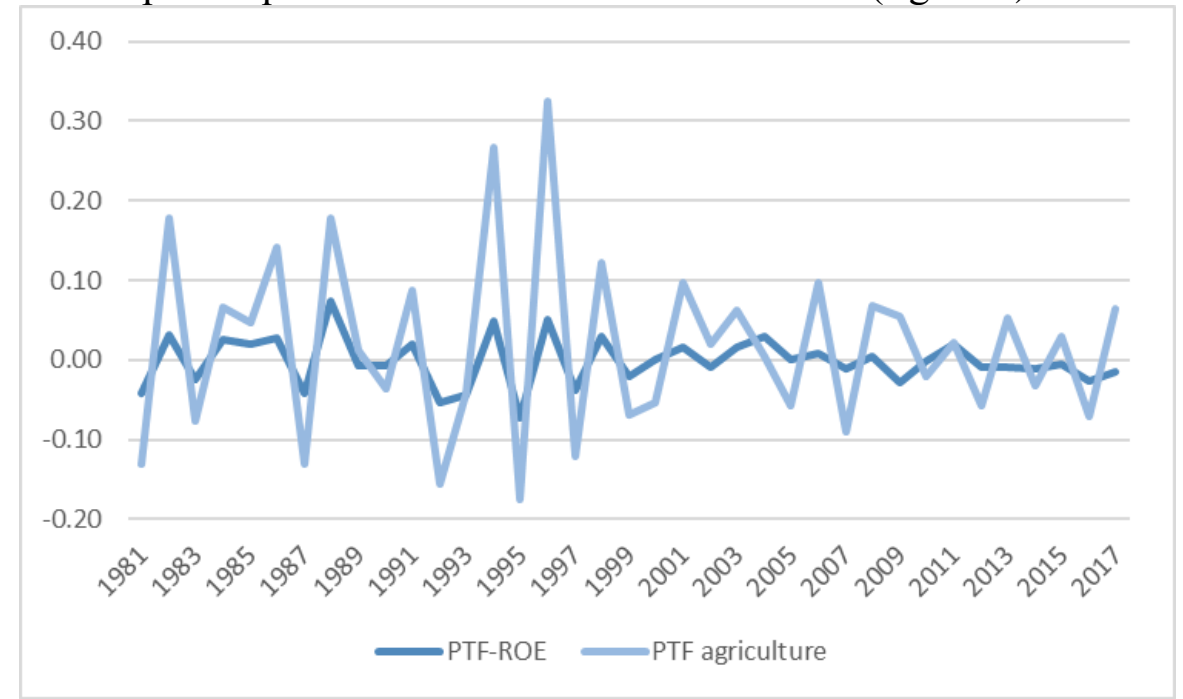

Figure 4 : Evolution de la PTF par secteur sur la période 1981-2017 Source : nos calculs

Même si la productivité totale des facteurs est plus importante dans le secteur agricole, celle-ci demeure très volatile par rapport aux autres secteurs (figure 4). L'analyse des séries des PTF montre que celle de l'agriculture se caractérise par un écart-type de l'ordre de 0,11, tandis que celui de la PTF des autres secteurs est de 0,03 . À partir de ces résultats, il est clair que les deux facteurs, capital et productivité totale des facteurs, jouent un rôle déterminant dans les niveaux de croissance agricole réalisés au Maroc. Ces deux facteurs sont étroitement liés aux orientations et décisions politiques retenues par les autorités publiques. En ce qui concerne le capital, le stock du capital économique dans une économie dépend principalement des investissements réalisés dans les différents secteurs tandis que l'amélioration de la productivité totale des facteurs est intimement liée aux politiques conduites (incitations, subventions, etc.). 
Suites à ces constats, une analyse plus approfondie de l'évolution de la PTF et de la croissance des autres facteurs de production par période a été réalisée. Ces périodes représentent les différentes politiques conduites dans le pays depuis 1980. Globalement, sur la période d'étude retenue (1980-2017), trois périodes durant lesquelles les pouvoirs publics ont massivement investi dans l'agriculture ont été retenues. La première période considère la phase de l'ajustement structurel. La deuxième consiste en la phase de l'après ajustement structurel et la dernière considère la phase de la planification sectorielle. Au niveau de cette section, les niveaux de performance agricole enregistrés sont expliqués par les instruments de politique adoptés pour atteindre les niveaux de productivité et de croissance espérés.

\section{Période d'ajustement structurel}

$\mathrm{Au}$ lendemain de son indépendance, le Maroc a conduit une politique de substitution aux importations capitalisant sur l'exportation des produits agricoles, sur une industrie de textile naissante et sur les revenus des phosphates. L'échec de cette politique interventionniste suite à plusieurs raisons (l'accentuation des disparités économiques et sociales; la chute des prix des phosphates sur le marché international ; l'accroissement de la dette et des déséquilibres économiques) a poussé les autorités publiques à mettre en place une réforme structurelle ayant pour base une vision plus libérale. D'abord, la réforme s'est traduite par une privatisation des secteurs publics productifs, en particulier le secteur industriel, et la réduction des dépenses dans plusieurs domaines notamment des équipements. En parallèle, un démantèlement des protections douanières quantitatives afin d'ouvrir le pays sur le marché international a été mis en place dans le but de moderniser les secteurs productifs et attirer plus de capitaux que ce soit nationaux ou étrangers. A l'exception du secteur agricole, les prix ont été également libérés, les subventions ont été réduites et les monopoles supprimés (Clément, 1995). Durant cette période 1980-1991, la croissance économique a été de 4\% tandis que celle du secteur agricole a été de $8 \%$, et celle des autres secteurs de 3,7\%. La croissance économique a été principalement tirée par le secteur agricole. En effet, à elle seule, l'agriculture a contribué à presque la moitié de la croissance économique enregistrée, soit $48 \%$.

Bien qu'elle ait enregistré des taux de croissance importants, la croissance agricole a été très volatile en raison des fréquences élevées des années de sécheresse, surtout en début de période (figure 5). En effet, la forte croissance observée de l'agriculture s'explique plus par les faibles niveaux de production en début de période, plutôt que par une augmentation moyenne de la production. Cette croissance a été majoritairement expliquée par la croissance du stock du capital qui a enregistré un taux de croissance de l'ordre de $7 \%$ alors que celui du travail ne dépassait pas les 0,2\% (tableau 5 et 6). 


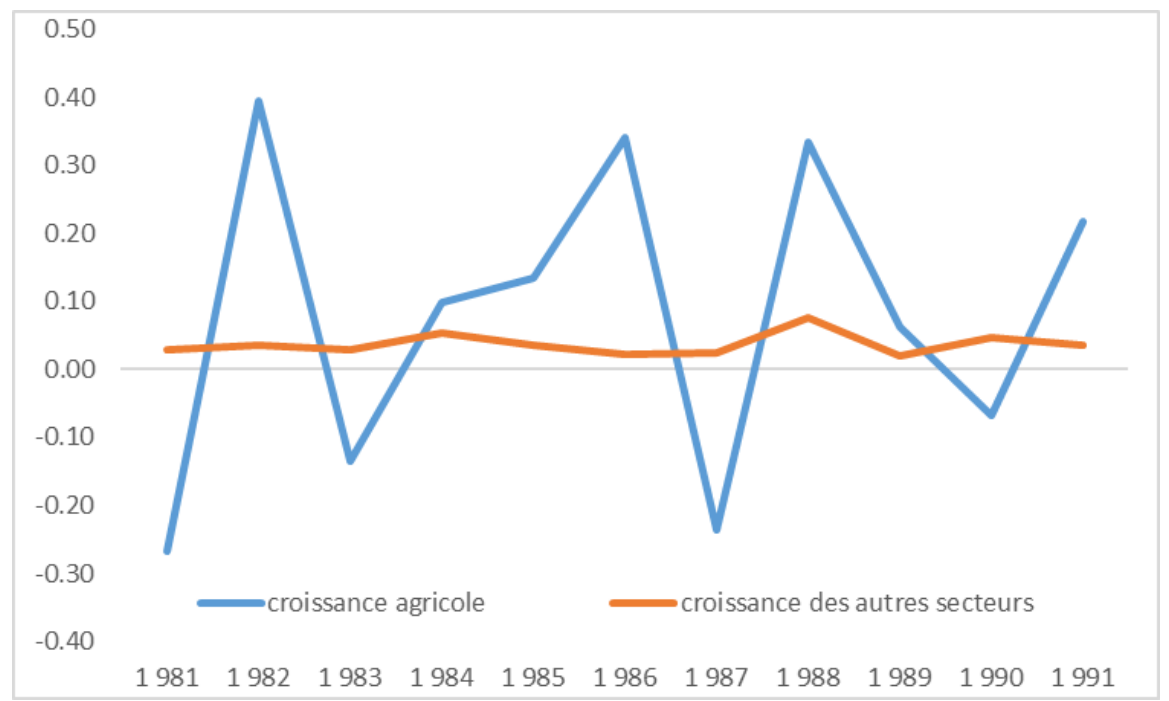

Figure 5. Taux de croissance agricole et des autres secteurs - entre 1981 et 1991

Durant cette période, l'Etat s'est engagé également dans de grands projets d'amélioration du secteur irrigué, ce qui s'est traduit par une augmentation de la consommation en eau d'environ $6 \%$. Ces projets ont visé l'amélioration de l'efficience de l'utilisation de l'eau à travers les deux grands programmes PAGI I et PAGI II.

Enfin, l'amélioration de la productivité a été nettement supérieure dans le secteur agricole comparé aux autres secteurs. En effet, celle-ci a augmenté de 3\% dans l'agriculture contre seulement $0,6 \%$ dans les autres secteurs (Tableau 5). Cela reflète une utilisation plus efficace et efficiente des ressources dans l'agriculture, notamment des ressources en eau. La modernisation des techniques de production et le soutien apporté aux agriculteurs dans le cadre de la politique des barrages ont permis une amélioration significative de la productivité totale des facteurs.

Tableau 5. Taux de croissance des valeurs ajoutées par secteurs, des quantités des facteurs de production utilisés, et de la productivité totale des facteurs sur la 1980-1991

\begin{tabular}{|c|c|c|c|c|c|c|}
\hline 1980-1991 & $\begin{array}{c}\text { Taux de } \\
\text { croissance }\end{array}$ & Eau & Terre & Travail & Capital & PTF \\
\hline Agriculture & 8,0 & 6,3 & 1,5 & 0,2 & 6,8 & 3,0 \\
\hline ROE & 3,7 & - & - & 3,3 & 2,3 & 0,6 \\
\hline
\end{tabular}


Tableau 6. Contribution des facteurs de production à la croissance sectorielle sur la période 1980-1991 en pourcent

\begin{tabular}{|c|c|c|c|c|c|}
\hline 1980-1991 & Eau & Terre & Travail & Capital & PTF \\
\hline Agriculture & 4 & 2 & 0,5 & 58 & 38 \\
\hline ROE & - & - & 23 & 59 & 18 \\
\hline
\end{tabular}

Source : nos calculs

\section{Période de post-ajustement structurel}

Après avoir fourni des efforts en termes de politiques d'ajustement structurel et de libéralisation sectorielle, la croissance économique au Maroc n'a pas été au rendez-vous. En effet, pendant cette période de l'après ajustement, le taux de croissance est passé de 4,2\% à environ 3\%. Cette croissance a été essentiellement tirée par la croissance du capital enregistrant une hausse de 4,5\%. Durant cette période, l'emploi a augmenté de 1,2\% et la PTF n'a augmenté que de 0,6\%. Les années de sécheresse qu'a connues le Maroc durant cette période ont fait baisser les niveaux d'eau stockée dans les barrages de façon significative. Suites à ces conditions critiques, les quantités d'eau lâchées ont baissé de $0,6 \%$ et les superficies cultivées ont baissé à leur tour de $0,3 \%$ (tableau 7 ).

Tableau 7. croissance des facteurs de production et de la PTF et leur contribution à la croissance observée en pourcent

\begin{tabular}{|c|c|c|}
\hline 1992-2006 & Croissance & Contribution \\
\hline Travail & 1.2 & 12 \\
\hline Capital & 4.5 & 79 \\
\hline Terre & -0.3 & -0.2 \\
\hline Eau & -0.6 & -0.2 \\
\hline PTF & 0.6 & 16 \\
\hline
\end{tabular}

Source : nos calculs

La phase de l'après ajustement structurel a été marquée par une croissance agricole erratique, puisque l'agriculture a connu de grandes fluctuations climatiques. Cependant, en moyenne le secteur de l'agriculture a enregistré une croissance annuelle moyenne relativement élevée en comparaison aux autres secteurs de l'économie, soit 5,8\%. L'essentiel de cette croissance est le résultat de l'augmentation du capital. En effet, celui-ci a contribué en moyenne par $74 \%$ à la croissance de la valeur ajoutée. Cette augmentation du capital traduit l'effet combiné à la fois des subventions importantes au capital et l'amélioration de la rentabilité relative dans le secteur grâce aux différentes mesures prises par le gouvernement notamment l'exonération fiscale et la dé-protection des autres secteurs de l'économie. En 
conséquence l'accumulation du capital s'est intensifiée et conjointement le travail a continué d'enregistrer une tendance baissière d'environ $-0,1 \%$, En ce qui concerne les ressources hydriques, en raison de mauvaises conditions climatiques, celles-ci ont baissé de façon significative durant cette période accusant un taux de croissance de l'ordre de -0,6\%. L'intensification du capital ne s'est accompagnée que d'une amélioration modérée de la PTF (1.68\%), comparativement à la période précédente. Cependant, la contribution de la PTF à la croissance dans le secteur $(27,23 \%)$ a permis de compenser pour le manque de contribution du travail à la croissance (tableau 8 et 9).

Tableau 8. Taux de croissance des valeurs ajoutées, des quantités de facteurs de production utilisées et de la PTF par secteur sur la période 1992-2006 en pourcent

\begin{tabular}{|c|c|c|c|c|c|c|}
\hline 1992-2006 & $\begin{array}{c}\text { Taux de } \\
\text { croissance }\end{array}$ & Eau & Terre & Travail & Capital & PTF \\
\hline Agriculture & 5,8 & $-0,6$ & $-0,3$ & $-0,1$ & 6,3 & 2,5 \\
\hline ROE & 3,8 & - & - & 2,4 & 4,9 & $-0,02$ \\
\hline
\end{tabular}

Tableau 9. Contribution des facteurs de production et de la PTF à la croissance sectorielle sur la période 1992-2006 en pourcent

\begin{tabular}{|c|c|c|c|c|c|}
\hline 1992-2006 & Eau & Terre & Travail & Capital & PTF \\
\hline Agriculture & -0.4 & -1 & 0 & 74 & 44 \\
\hline ROE & - & - & 22 & 84 & $-0,07$ \\
\hline
\end{tabular}

Source : nos calculs

\section{Période des stratégies sectorielles}

Confronté à des taux de croissance très en dessous de ceux espérés par la mise en application de la politique de libéralisation et d'ajustement structurel, et face aux nouveaux défis de la globalisation, le Maroc a adopté une nouvelle stratégie de développement économique et social basée sur les plans sectoriels. Ces plans ont en commun une politique volontariste d'encouragement à l'investissement privé national et étranger et une plus grande volonté de libéralisation de l'économie nationale. Cette dernière s'est traduite par une série d'accords bilatéraux et multilatéraux de libre-échange (Union européenne, USA, Accord d'Agadir, Accord avec la Turquie), création de zones franches, plus grande flexibilité dans la politique de contrôle de change.

Cette période a été marquée par une croissance économique de l'ordre de 3,6\%. Cette croissance a été principalement tirée par l'investissement dans le capital qui a vu son stock augmenter de 5,6\%. En parallèle, le travail a enregistré une hausse de seulement de $0,8 \%$ contribuant ainsi à environ $8 \%$ du PIB. Enfin, la PTF a augmenté de $0,1 \%$ et sa contribution à la création de 
richesse a été de $2,6 \%$, soit la contribution la plus faible dans toute la période étudiée.

Au niveau sectoriel, l'agriculture demeure le moteur de la croissance économique avec un taux de croissance annuelle moyenne de 4,6\%. Ayant bénéficié de multiples programmes d'investissements publics et de subvention à l'investissement privé, le capital a connu une croissance annuelle moyenne de $6,5 \%$, contribuant ainsi à $63 \%$ de la croissance dans le secteur. En effet, sur la période 2008-2017, le cumul des investissements public (hors incitations octroyées au secteur privé a été de l'ordre de 43 milliards de dirhams tandis que le cumul des investissements privés a été estimé à 56 milliards de dirhams. La ventilation des incitations à l'investissement privé, qui se sont principalement inscrits dans le cadre des subventions accordées par le fonds de développement agricole, montre que sur la période 2008-2017, la majeure partie des incitations ont concerné le secteur de l'irrigation. En effet, $47 \%$ des subventions ont été accordées pour l'introduction du système d'irrigation localisée et l'aménagement des terres agricoles inscrits dans le cadre du Programme National d'économie l'eau d'irrigation (PNEEI), tandis que 21\% ont été accordés à l'équipement agricole.

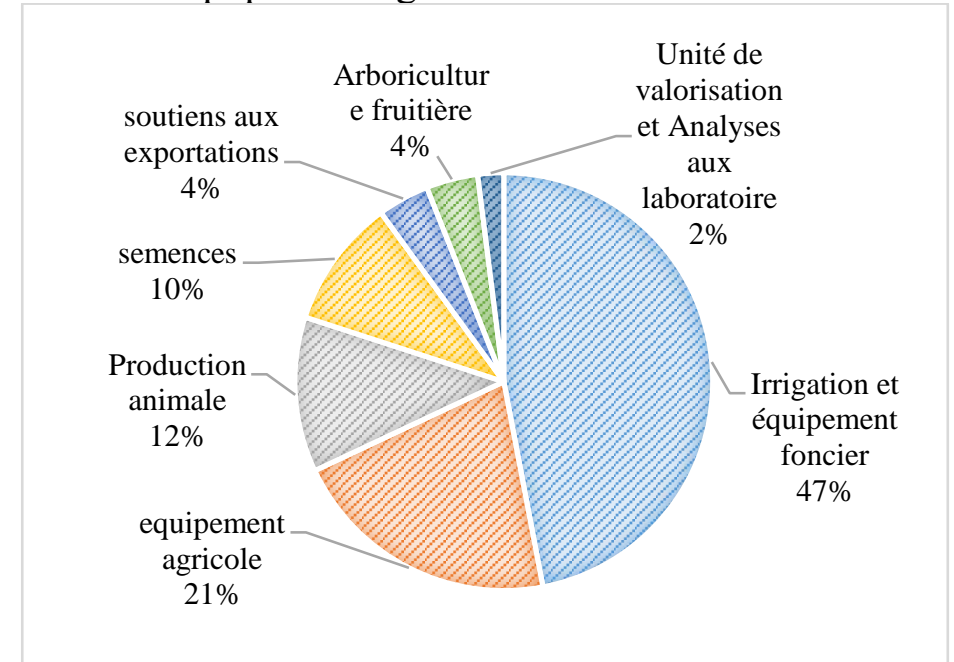

Figure 6. Structure de subvention du FDA à l'investissement privé sur la période 2008-2017 Source : DEPF, 2019

La promotion de l'irrigation a été naturellement accompagnée par une intensification de production au niveau des périmètres irrigués. A cet effet, l'utilisation des eaux dans le secteur agricole a connu une hausse de 3,9\%. Cette augmentation de quantités d'eau d'irrigation a permis d'expliquer environ $4 \%$ de la croissance agricole. En parallèle, l'agriculture a continué de libérer la main d'œuvre. La croissance de la population active employée dans le secteur agricole a baissé de 1,2\%. Cette baisse est majoritairement expliquée 
au recours à l'utilisation des machines et engins agricoles dont la demande a augmenté grâce aux subventions débloquées par l'Etat et des différentes facilitations accordées dans le cadre du Plan Maroc Vert. La baisse du nombre des travailleurs agricoles a permis à l'agriculture d'améliorer sa productivité. En effet, l'amélioration de la productivité a été de $1 \%$, contribuant ainsi à plus de $14 \%$ de la croissance agricole observée (tableaux 9 et 10).

Notons que cette période a été également marquée par plusieurs contraintes, notamment la crise économique de 2007 qui s'est traduite par une demande notamment en produits agricoles extérieure plus réduite. Malgré cet environnement économique défavorable, l'agriculture a continué d'enregistrer les meilleures performances en termes d'amélioration de la productivité comparée aux autres secteurs.

Tableau 10. Taux de croissance des valeurs ajoutées, de l'utilisation des facteurs de production et de la PTF par secteur sur la période 2007-2017 en pourcent.

\begin{tabular}{|c|c|c|c|c|c|c|}
\hline 2007-2017 & $\begin{array}{c}\text { Taux de } \\
\text { croissance }\end{array}$ & Eau & Terre & Travail & Capital & PTF \\
\hline PIB & 3,9 & 3,9 & 0,5 & 0,8 & 5,6 & 0,1 \\
\hline Agriculture & 4,6 & 3,9 & 0,5 & $-1,2$ & 6,5 & 1,0 \\
\hline ROE & 3,5 & & & 2,1 & 5,5 & $-0,7$ \\
\hline
\end{tabular}

Tableau 11. Contribution des facteurs de production et de la PTF à la croissance sectorielle sur la période 2007-2017 en pourcent

\begin{tabular}{|c|c|c|c|c|c|}
\hline 2007-2017 & Eau & Terre & Travail & Capital & PTF \\
\hline PIB & 1.1 & 0.3 & 7.9 & 96.9 & 2.6 \\
\hline Agriculture & 4 & 1,2 & -4.2 & 96,3 & 21 \\
\hline ROE & - & - & 21 & 102 & -23 \\
\hline
\end{tabular}

\section{Conclusion}

Source : nos calculs

La décomposition de la croissance observée par contribution des facteurs de production à savoir l'eau, la terre, le capital et le travail a montré que l'économie marocaine est principalement tirée par le capital. Ce dernier a augmenté durant la période étudiée de $6,2 \%$ contribuant ainsi en moyenne à environ de $68 \%$ à la croissance du PIB national. Quant au travail, sa contribution demeure faible de l'ordre de $9 \%$, témoignant ainsi d'un approfondissement du capital dans l'économie « capital deepening » que d'un élargissement du capital « capital widening ».

Grâce aux multiples efforts consentis par l'Etat en termes de développement du secteur de l'eau, la croissance de l'utilisation des ressources hydriques à des fins agricoles a été de $3 \%$ contribuant ainsi à $0,7 \%$ de la croissance du revenu national et $2,1 \%$ de la croissance de la valeur ajoutée 
agricole. L'irrigation demeure un facteur décisif quant aux niveaux de performances enregistrés non seulement par sa contribution significative à la croissance mais aussi par sa participation à l'amélioration de la productivité totale des facteurs. En effet, l'analyse de la PTF a montré que sur les trois périodes étudiées (1980-1991, 1992-2006, 2007-2017), le secteur agricole a enregistré les niveaux d'amélioration de productivité les plus élevés. Certes durant la phase d'ajustement structurel, et en raison des mesures de libéralisation de l'économie marocaine, la rentabilité relative de l'agriculture s'est améliorée, ce qui a attiré plus d'investissement dans le secteur (croissance du capital d'environ 7\%). Toutefois, durant cette période, l'Etat s'est engagé également dans de grands projets d'amélioration du secteur de l'irrigation, ce qui s'est traduit par une augmentation de la consommation en eau d'environ $6 \%$ et une amélioration de la productivité d'environ 3\%. Ces projets ont visé l'amélioration de l'efficience de l'utilisation de l'eau à travers les deux grands programmes PAGI I et PAGI II. Durant la période suivante, le Maroc a été frappé par plusieurs vagues de sécheresse. Toutefois, le secteur agricole a continué d'enregistrer des taux de croissance assez élevés. Suite aux conditions climatiques défavorables, l'utilisation des eaux dans l'irrigation a baissé d'environ $0,6 \%$, parallèlement, l'amélioration de la productivité a été très significative, enregistrant un taux de croissance moyen de l'ordre de 2,5\% et contribuant à $44 \%$ de la croissance du secteur agricole. Durant cette période, le secteur de l'eau a subi plusieurs changements, notamment juridique par l'introduction de la nouvelle loi sur l'eau en 1995, qui est venue clarifier le statut de l'eau et les conditions d'utilisation de cette ressource. Elle a aussi insisté sur la gestion intégrée des ressources en eau pour une meilleure utilisation de celle-ci.

La dernière phase a été marquée par une intervention massive de l'Etat en termes de promotion de nouvelles techniques d'irrigation plus économes en eau dans le cadre du PNEEI. Suite aux différentes incitations dédiées à l'irrigation, l'utilisation de l'eau a augmenté de $4 \%$ en raison de l'intensification de la production et l'orientation vers les cultures plus rentables et à la fois plus exigeantes en termes d'eau. Ainsi, l'augmentation des volumes destinés à l'irrigation a contribué à la formation de $2,5 \%$ de la valeur ajoutée agricole. La croissance de la PTF a été positive contribuant ainsi à plus de $14 \%$ de la valeur ajoutée agricole et le capital a continué de jouer un rôle primordial dans la croissance agricole observée.

\section{References:}

1. Acemoglu, D. (2002). Directed Technical Change. The Review of Economic Studies, 69(4), 781-809. 
2. Acemoglu, D., Johnson, S., \& Robinson, J. (2004). Institutions As The Fundamental Cause Of Long-Run Growth. NBER Working Paper Series (10481).

3. Ahmed, Z. (2008). Dépenses Publiques Et Croissance Economique Quel Rôle Pour L'Etat? Revue d'économie et de statistique appliquée. Volume 5, Numéro 1, Pages 115-132

4. Agoumi, A., (2006). Ressources en eau et bassins versants du Maroc : 50 ans de développement (1955-2015). Maroc : IRES.

5. Aspe, C., (2012). De l'eau agricole à l'eau environnementale : résistance et adaptation aux nouveaux enjeux de partage de l'eau dans les pays du bassin méditerranéen. Paris: Update sciences \& technologies.

6. Baldwin, J. (2005). Estimating Depreciation Rates for the Productivity Accounts. Ottawa, Canada : Micro-Economics Analysis Division. Statistics Canada.

7. Bouderbala, N., (1999). L'aménagement des grands périmètres irrigués : l'expérience marocaine. Cahiers Options Méditerranéennes (36), 171184.

8. Bu, Y. (2004). Fixed Capital Stock Depreciation in Developing Countries: Some Evidence from Firm Level Data. Liberty Mutual Group, Boston, USA.

9. DEPF. (2019). Le secteur agricole marocain : Tendances structurelles, enjeux et perspectives de développement. Direction des Etudes et des Prévisions Financières, 1-30.

10. DEPF. (2020). Le Maroc à l'épreuve du changement climatique : situation, impacts et politiques de réponse dans les secteurs de l'eau et de l'agriculture. Policy brief $\mathrm{n}^{\circ} 18$. Direction des Etudes et des Prévisions Financières. Royaume du Maroc

11. Doukkali, M. R., (2005). Water institutional reforms in Morocco. Water Policy, 7(1), 71-88.

12. Doukkali, M. R., (2010). Matrice de comptabilité sociale : cas du Maroc. Département des sciences humaines. Institut Agronomique et Vétérinaire Hassan II.

13. Doukkali, M., \& Grijsen, J., (2018). Evaluation de la Contribution Economique de la Surexploitation des Eaux Souterraines dans l'Agriculture au Maroc. Policy Center for the New South.

14. Ezzahra Mengoub F., Lejars C. \& Rachid Doukkali M. (2021). Impacts De La Politique De Reconversion Des Systèmes D'irrigation Gravitaire vers l'irrigation Localisée : Cas Du Sous Bassin Du Tadla Azilal. European Scientific Journal, ESJ, 17(24), 46. 
15. Harbouze R., Pellissier J.-P., Rolland J.-P., Khechimi W. (2019). Rapport de synthèse sur l'agriculture au Maroc. [Rapport de recherche] CIHEAM-IAMM. pp.104. ffhal-02137637v2f

16. Hssaisoune, M., Bouchaou, L., Sifeddine, S., Bouimetarhan, I., Chehbouni, A. (2020). Moroccan Groundwater Resources and Evolution with Global Climate Changes. Geosciences 2020, 10, 81; doi:10.3390/geosciences 10020081

17. HCP. (2005). Les sources de croissance au Maroc. Haut-Commissariat au Plan. Royaume du Maroc.

18. Huard, P. (1971). Tour d'horizon : programmation non linéaire. Revue française d'informatique et de recherche opérationnelle, série rouge, tome 5, no 1 (1971), p. 3-48.

19. Kleiche, M., (2001). Aux Origines Du Concept De Développement. Quand L'Irrigation Devient Enjeu De Réforme Agricole : Nouvelle Mise En Ordre Du Paysage Rural Marocain Dans L'Entre-DeuxGuerres. Hespéris-Tamuda, 175-194.

20. Nehru, V., \& Dhareshwar, A. (1993). A New Database On physical Capital Stock: Sources, Methodology and Results. Revisita De Analysis Economico, Vol. 8( $\left.\mathrm{N}^{\circ} 1\right), 37-59$.

21. MAPMDREF (2013). Fonds de Développement Agricole : Les Aides Financières de l'État pour l'encouragement des investissements agricoles. Rapport du Ministère d'agriculture, de la pêche maritime, du développement rural et des eaux et forêts. Royaume du Maroc.

22. MEME, (2010). Rapport National sur l'Etat de l'Environnement. Ministre de l'Energie, des Mines et de l'Environnement. Gouvernement du Maroc.

23. Mili, E. (2020). Intégration du genre dans la gestion des ressources en eau au Maroc (Nord-Ouest d'Afrique). 20th \& 21st Century French and Francophone Studies International Colloquium. University of Nebraska - Lincoln

24. Molle, F., Sanchis-lbor C.; Avellà-Reus, L. (2019). Irrigation in the Mediterranean Technologies, Institutions and Policies. Chapter 2:

25. Morocco. Global Issues in Water Policy. Springer Nature Switzerland AG 2019. Hardcover ISBN: 978-3-030-03696-6

26. North, D. C., (1990). Institutions, Institutional Change and Economic Performance. New York: Cambridge University Press.

27. OCDE (2009). Measuring Capital OECD Manual. Second Edition: Organisation for Economic Co-Operation And Development.

28. Roe, T., \& Smith, R. (2014). MONOGRAPH: Introduction to Growth Accounting as a Diagnostic Tool. Department of Applied Economics. University of Minnesota. 
29. Taheripour, F., Wallace E. T., Iman H., and Ehsanreza S. (2020). "Water Scarcity in Morocco: Analysis of Key Water Challenges." World Bank, Washington, DC.

30. SECEE. (2011). Projet de Renforcement des Capacités sur l'Utilisation sans danger des Eaux Usées en Agriculture. Maroc : Secrétariat d'Etat auprès du Ministère de l'Energie, des Mines, de l'Eau et de l'Environnement chargé de l'Eau et de l'Environnement.

31. Sadiki, M (2017). La rareté de l'eau : défis et opportunités - cas du secteur agricole au Maroc - Séminaire de haut-niveau, « rareté de l'eau : défis et opportunités ». Novembre 2017. Rome, Italie.

32. Solow, R. M. (1956). A Contribution to the Theory of Economic Growth. The Quarterly Journal of Economics, 70(1), 65-94.

33. Welhan, K. (2020). Growth Accounting. University College Dublin, Advanced Macroeconomics Notes. P1-20

34. WRI, (2019). 17 Countries, Home to One-Quarter of the World's Population, Face Extremely High Water Stress. World Resources Institute. https://www.wri.org/insights/17-countries-home-onequarter-worlds-population-face-extremely-high-waterstress?fbclid=IwAR2 wrpvl83TNV0dIcFswDbixTu2qDtJA71cnbJfUTe03ueiLnMv1-AdMrs

35. Zaman, G., \& Goschin, Z. (2010). Technical change as exogenous or endogenous factor in the production function models. empirical evidence from Romania. Romanian Journal of Economic Forecasting, $29-45$. 